\title{
Performance Evaluation of Priority Load-Aware Scheduling (PLAS) Algorithm for IEEE802.16 Networks
}

\author{
Abdulhakeem Abdulazeez, Solomon Orduen Yese, and Aminu Mohammed
}

\begin{abstract}
In recent years, there has been an increase in the transmission of multimedia services due to the emergence of wireless broadband (WiBB) technologies such as WiMAX. WiMAX supports multiple QoS classes for transmission of different multimedia applications. However, satisfying the requirements of these applications with the often-limited resources has been a major challenge, which requires an efficient scheduling scheme such as PLAS. PLAS is a variant of LAWRR designed to reduce queuing delay of real-time traffics in WiBB networks. Several simulation experiments were conducted to evaluate the performance of PLAS against the LAWRR algorithm. The results revealed that PLAS outperforms the other scheme in terms of average delay for low input traffic. However, the metric (delay) used and the traffics generated for the simulation are not adequate to realistically evaluate the performance of a scheduling algorithm in a typical metropolitan area network. In this study, we further evaluated the performance of PLAS and LAWRR under varying higher input traffics, and an additional performance metric, using discrete event simulation. The results demonstrated that the PLAS achieved better results in terms of queuing delay and throughput compared to LAWRR. The improved performance will lead to better user experience, which will increase the number of subscribers and consequently increase revenue for service providers.
\end{abstract}

Index terms-WRR, PLAS, Scheduling algorithm, WiMAX,
QoS

\section{INTRODUCTION}

Recently, Wireless broadband (WiBB) technology have undergone tremendous advancement which has led to increased demand for high speed transmission of multimedia services over long distances. IEEE 802.16 also known as Worldwide Interoperability for Microwave Access (WiMAX) is a WiBB technology that was aimed at providing last mile internet access that can blanket up to $15 \mathrm{~km}$ and $50 \mathrm{~km}$ radius for mobile and fixed stations respectively [1]. Apart from its ease and cost effective infrastructure, WiMAX provides QoS support for different traffic types as follows:

UGS class defines fixed-sized data packets that require constant bit rate (CBR) transmission such as E1/T1 lines. The class characterizes real-time or high priority applications. rtPS class is designed to support variable sized packets that are generated intermittently. The services in this class are define by minimum reserved traffic rate, maximum sustained traffic rate and maximum latency.

Published on November 27, 2019.

Abdulhakeem Abdulazeez, Computer Science Unit, Department of Mathematics, Usmanu Danfodiyo University, Sokoto, Nigeria.

(email: Abdulhakeem.abdulazeez@udusok.edu.ng)

Solomon Orduen Yese, Computer Science Unit, Department of

Mathematics, Usmanu Danfodiyo University, Sokoto, Nigeria.

(email: yese.solomon@udusok.edu.ng)

Aminu Mohammed, Computer Science Unit, Department of Mathematics, Usmanu Danfodiyo University, Sokoto, Nigeria.

(email: mohammed.aminu@udusok.edu.ng)
rtPS supports applications such as MPEG video conferencing and streaming [3][6 - 8].

nrtPS class is designed for non-real-time traffics that do not require delay guarantee. The applications supported by this service are delay tolerant, require minimum bandwidth

and consists of variable-sized data packets. Example of such applications is the File Transfer Protocol (FTP) [3] [6 8].

BE class supports for applications such as Hypertext Transport Protocol (HTTP) and electronic mail (e-mail), that do not require QoS guarantee. These applications are defined by their need for maximum sustained traffic rate, traffic priority and request/transmission policy [3][6 - 8].

Each of these classes has unique QoS requirement in terms of delay, jitter, throughput and packet loss. Therefore, sharing the often scares network resources among the classes require an efficient scheduling algorithm.

Scheduling is a mechanism used to distribute available network resources among contending subscriber stations. WiMAX standard did not define any scheduling scheme for its resource management, which is the reason many researches [2] [4] [5] [7] [9-13] have been carried out to provide an efficient scheme that can efficiently share these scare network resources.

Recently, a Priority Load-Aware Scheduling (PLAS) algorithm was proposed to reduce the problem of delay in LAWRR [2]. The PLAS separates traffics according to their QoS requirements. It assigns a dynamic weight to each class at the beginning of every counter reset. The weight prioritizes traffics by increasing the service rate of real-time traffics over other traffics.

The performance of the PLAS algorithm was evaluated against LAWRR only in terms of average delay under varying network sizes of 5,10,15,20,25,30 and $35 \mathrm{SSs}$, and the results revealed that PLAS outperforms LAWRR. However, the traffics generated by these SSs are not bursty enough to realistically evaluate the performance of PLAS in a typical metropolitan network environment. Moreover, there is need to determine its performance based on other QoS requirements like average throughput, packet loss, jitter, and latency.

In this paper, we present performance evaluation of PLAS against LAWRR in terms of average delay and throughput under more bursty traffics environment.

The rest of this paper is organized as follows: In Section II, a brief review of some existing works is presented; Section III presents the operation PLAS algorithm; Section IV presents performance evaluation. Finally, Section V concludes the paper.

\section{EXISTING SCHEDULING AlgORITHMS}

This section presents a brief review of some existing scheduling algorithms in WiMAX as follows: 


\section{A. Round Robin Algorithm}

In [9] round-robin (RR) algorithm was proposed to classify traffics into different classes based their priorities. The RR serves all nonempty queues in a cyclic fashion, starting from high to low priority queues. The algorithm allocates the same bandwidth to all nonempty queues at the beginning of a counter reset. Therefore, it is fair for traffics with the same cost, but fail provide QoS guarantee for different service classes.

\section{B. Weighted Round Robin}

Authors in [10] proposed weighted round-robin (WRR) algorithm to improve RR algorithm by providing QoS guarantee for each service class. The WRR starts by separating queues according to their priorities. Then, it assigns a static weight to the weight counter of each queue. The weights determine the number of packets that can be served from each queue in each service round. The value of the weight counter is decremented by one each time a packet is served from a queue until the weight value becomes zero or when there are no more packets in the queue. Therefore, the algorithm skips all empty queues until after the next counter reset when new weights will be assigned. WRR is fair when all the queues have equal packets sizes. However, it will cause increase in delay, packet loss and decrease in average throughput under busty traffics arrival rate due to its failure to prioritize traffic according to their QoS requirements.

\section{Modified Weighted Round Robin}

In [11], a modified weighted round robin (MWRR) scheduling algorithm was proposed to reduce delay in WRR. The MWRR algorithm allocates weights to all nonempty queues according to their QoS requirements. The weight is computed as a product the static WRR and a constant multiplier value to increase the increase the service rate of each queue. The multiplier is an integer value obtained according to the network size. The value is large for smaller network and vice versa. The MWRR reduces average delay but will cause increase in average delay and decrease in average throughput if the multiplier is inappropriately obtained.

\section{Adaptive Weighted Round-Robin}

Authors in [12] proposed another variant of WRR called adaptive weighted round-robin (AWRR) scheduling algorithm to improve the performance of WRR in terms of delay. The AWRR uses two schedulers; input and output to serve packets from the service classes. The input scheduler prioritizes real-time traffics over other traffics according to their QoS requirements, while the output scheduler regulates data flow from the service classes. The algorithm adjusts the weight of each class when a threshold set for the class is reached. AWRR reduces delay and increases average throughput. However, it causes increase in average delay, latency, packet loss and decrease in average throughput if the threshold value is wrongly assigned.

\section{E. Latency Weighted Round Robin}

In [13] a low latency weighted round robin (LLWRR) scheduling algorithm is proposed to reduce latency of real time traffics in AWRR. The LLWRR assigns weight to all nonempty queues at the beginning of every counter reset. The weight is obtained as a product of the static WRR weight and a dynamic coefficient value. The coefficient value is an integer number obtained according to number of queues. The value decreases as the number of queues increases and vice versa. LLWRR improves fairness and decreases latency. However, it causes increase in delay and packet drop under high input traffic network with large number of queues, due to the decrease in size of the multiplying coefficient.

\section{F. Load-Aware Weighted Round Robin}

A load-aware weighted round-robin (LAWRR) algorithm was proposed in [2] to improve WRR. The LAWRR separates packets into different service queues according to their QoS requirements. It assigns weight to each queue at the beginning of every counter reset. The weight is computed based on the queue's load characteristics, by multiplying dynamic coefficient of the queue and a static WRR weight. The algorithm serves packet from all nonempty queue in RR fashion It reduces average delay and packet loss as well as improves average throughput. However, it causes increase in delay because of its failure to prioritize traffics. It also increases packet drop rate and decreases average throughput in real time traffics due to its lack of considerations for packet deadline.

\section{G. Class-Based QoS Scheduling Algorithm}

In [15], a class-based QoS scheduling (CBS) is proposed to improve performance of existing WiMAX scheduling algorithms in terms of throughput. The CBS separates QoS classes into two service groups-namely, delay constraint service (DCS) and throughput guarantee service (TGS). The DCS comprise of delay sensitive traffics while the TGS comprises of delay tolerant traffics. The scheme serves packets using a three-stage process: priority elevation (PE), virtual ranking queue (VRQ), and packet scheduler (PS). Weighted high priority (WHP) are assigned a service rate of $60 \%$ and $40 \%$ for weighted low priority (WLP). The PE classifies incoming packets into either WHP or WLP according to their deadlines. WHP holds packets with earlier deadlines, the packets are moved to VR and served using PS. The scheme elevates packets with earlier deadlines in WLP to VRQ to reduce drop rate. However, the scheme will cause an increase in packet drop of DCS under heavy input traffic arrivals as a result of frequent service interruption by WLP packets.

\section{PLAS ALGORITHM}

This section describes operation PLAS algorithm in [2]. The algorithm was proposed to address the performance degradation of real time traffics in LAWRR. It adaptively serves packets from each QoS queue using a modified priority weight defined as:

$$
M_{i, r}^{w}=P_{i, r}^{v} \cdot W_{i, r}^{d} \text {. }
$$

where $P^{v}{ }_{i, r}$ is the priority value of queue $i$ at round $r$ and $W^{d}{ }_{i, r}$ is the LAWRR weight of queue $i$ at round $r$.

The modified weight in Equation (1) prioritizes real time traffics by increasing their LAWRR transmission rate by 100 $\%$ if the number of packets in the queue is less than or equal to half size of the buffer. However, if the number of packets exceeds half of the buffer, then modified weight becomes the 
queue length. The algorithm maintains the LAWRR weights for non-real time traffics.

Fig. 1 and TABLE I are used to demonstrate the operation of the PLAS. The table shows that the modified weights are computed and represented as: $M w_{1,1}=4, M w_{2,1}=4, M w_{3,1}$ $=2$, and $M w_{4,1}=6$. These weights are assigned to respective weight counters as: $W C_{1,1}=4, W C_{2,1}=4, W C_{3,1}=2$, and $W C_{4,1}=6$ as shown in Fig.1. This Figure schedules packet as:

$$
\mathrm{q} 1,1 \rightarrow \mathrm{q} 1,2 \rightarrow \mathrm{q} 1,3 \rightarrow \mathrm{q} 1,4 \rightarrow \mathrm{q} 2,1 \rightarrow \mathrm{q} 2,2 \rightarrow \mathrm{q} 2,3 \rightarrow \mathrm{q} 2,4
$$
$\rightarrow \mathrm{q} 3,1 \rightarrow \mathrm{q} 3,2 \rightarrow \mathrm{q} 4,1 \rightarrow \mathrm{q} 4,2 \rightarrow \mathrm{q} 4,3 \rightarrow \mathrm{q} 4,4 \rightarrow \mathrm{q} 4,5 \rightarrow \mathrm{q} 4,6$

TABLE I: PLAS PRIORITY WEIGHT COMPUTATION

\begin{tabular}{cccccc}
\hline \hline $\boldsymbol{q}_{\boldsymbol{i , \mathbf { 1 }}}$ & $\boldsymbol{N}_{\boldsymbol{i , \mathbf { 1 }}}^{\boldsymbol{q}}$ & $\boldsymbol{W}^{\boldsymbol{d}}{ }_{\boldsymbol{i , \mathbf { 1 }}}$ & $\boldsymbol{P}^{\boldsymbol{v}}{ }_{\boldsymbol{i , \mathbf { 1 }}}$ & $\boldsymbol{M}_{\boldsymbol{i , \mathbf { 1 }}}^{\boldsymbol{w}}$ \\
\hline$q_{1,1}$ & 4 & 2 & 2 & 4 \\
$q_{1,2}$ & 5 & 4 & $5 / 4$ & 5 \\
$q_{1,3}$ & 3 & 2 & 1 & 2 \\
$q_{1,4}$ & 6 & 6 & 1 & 6 \\
\hline \hline
\end{tabular}

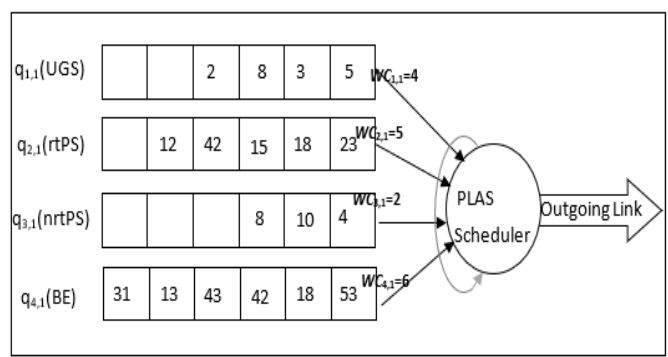

Fig. 1. The State of PLAS After Weights Are Assigned

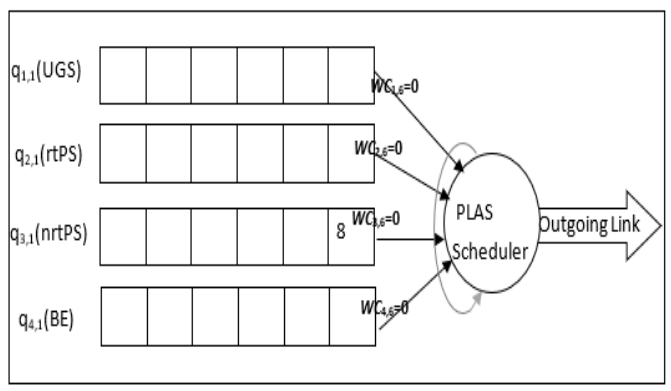

Fig. 2. The PLAS After Weights Have Been Exhausted

After packets are scheduled in Fig.1, Fig. 2 shows only one packet queued before the next counter reset. The PLAS compared with LAWRR in [14], demonstrated better performance in terms of delay under low to moderate traffic loads from 5,10,15,20,30 and $35 \mathrm{SSs}$ for different experiments. However, the metric (delay) used and the traffics generated by the SSs are not enough to realistically evaluate the performance of PLAS in a typical metropolitan area network. Thus, the need for further evaluation.

\section{PERFORMANCE EVALUATION}

Discrete event simulation was used to evaluate the performance of PLAS and LAWRR.

The simulation scenario and performance metrics used are presented in this section.

\section{A. Simulation model}

The simulation topology used as shown in Fig. 3, consists of one Base Station (BS), multiple Subscriber Stations (SSs) distributed around the BS, and an application server. The traffics are generated from the server, which provides four traffics each from a different application. We assume that each user traffic is carried by one SSs and that each user can only use one type of traffic in a given time. The traffics are prioritized according to their QoS requirements in the following order:

$\mathrm{UGS} \rightarrow \mathrm{rtPS} \rightarrow \operatorname{nrtPS} \rightarrow \mathrm{BE}$

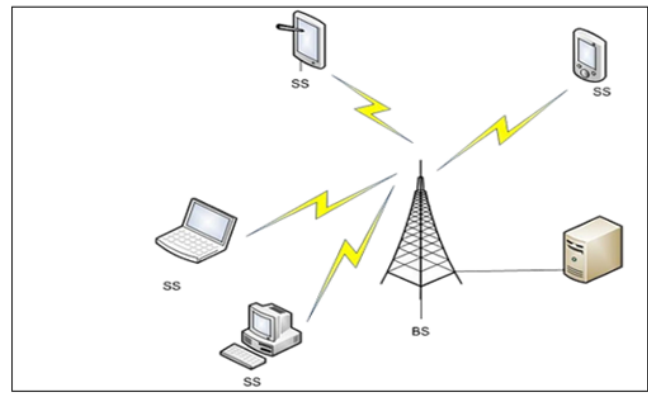

Fig. 3. Simulation Topology [14]

In this experiment, 20, 40, 60, 80, 100 and $120 \mathrm{SSs}$ are used for different simulation scenarios to evaluate the effect of increase in number of SSs on performance of proposed and benchmark algorithms. The simulation parameters in Table I,II and III are used.

TABLE II: SIMULATION PARAMETER [1] [9]

\begin{tabular}{ll}
\hline \hline Parameters & Values \\
\hline BS Frequency & $2.5 \mathrm{GHz}$ \\
Duplexing mode & TDD \\
System bandwidth & $5 \mathrm{Mbps}$ \\
DL/UL ratio & $2: 1(29: 18$ OFDM Symbol) \\
Frame Length & $5 \mathrm{~ms}$ \\
Cyclic prefix duration & $11.43 \mu \mathrm{s}$ \\
Basic Symbol & $91.43 \mu \mathrm{s}$ \\
FFT & 1,024 \\
PHY & OFDMA \\
DL permutation & PUSC \\
MAC PDU length & Variable \\
Fragment & Enable \\
ARQ and packing & Disable \\
DL - UL MAPs & Variable \\
\hline \hline
\end{tabular}

TABLE III: VIDEO TRAFFIC PARAMETERS [1][9]

\begin{tabular}{lll}
\hline \hline Parameters & Distribution & Values \\
\hline ON period & Exponential & Mean 1.34 s \\
OFF period & Exponential & Mean 1.67 s \\
Packet size & Constant & $66 \mathrm{~B}$ \\
IAT & Constant & $20 \mathrm{~ms}$ \\
\hline \hline
\end{tabular}

TABLE IV: VIDEO STREAMING PARAMETERS [1] [9]

\begin{tabular}{ll}
\hline \hline Parameters & Value \\
\hline Video packet size & Geometric (mean=200B) \\
Average traffic rate & $220 \mathrm{Kbps}$ \\
MRTR & $64 \mathrm{Kbps}$ \\
MSTR & $400 \mathrm{Kbps}$ \\
Maximum latency & $180 \mathrm{~ms}$ \\
Tolerated packet loss & 5 \\
IAT & Exponential $($ mean $=220 \mathrm{Kbps})$ \\
\hline \hline
\end{tabular}




\section{B. Performance Metric}

Two metrics; average delay and throughput were used to evaluate the performance of the PLAS and LAWRR scheduling algorithms.

Average delay: The mean waiting time in milliseconds by all packets in all the queues expressed as:

$$
A_{d}=\frac{1}{k} \sum_{i=1}^{k}\left[\frac{1}{N q_{i}} \sum_{j=1}^{N q_{i}}\left(A_{j}^{t}-D_{j}^{t}\right)\right]
$$

where $A^{t}$ is the packet arrival time, $D^{t}$ packet departure time, $N q_{i}$ is the number of packets in queue $i$, and $\mathrm{k}$ is the number of queues in the system.

Average throughput: The number of packets served by the scheduler per unit time in kilobits per seconds is expressed as:

$$
A^{t h}=\frac{1}{t} \sum_{i}^{n}\left(N q_{i}-A q_{i}\right)
$$

where $N q_{i}$ is the number of packets queued in queue $i$ within time $t, A q_{i}$ is the number of packets in queue $i$ after time $t, t$ is the total simulation time of the experiment, and $n$ is the number of queues.

\section{Simulation Results}

This section presents the results of the simulations as follows:

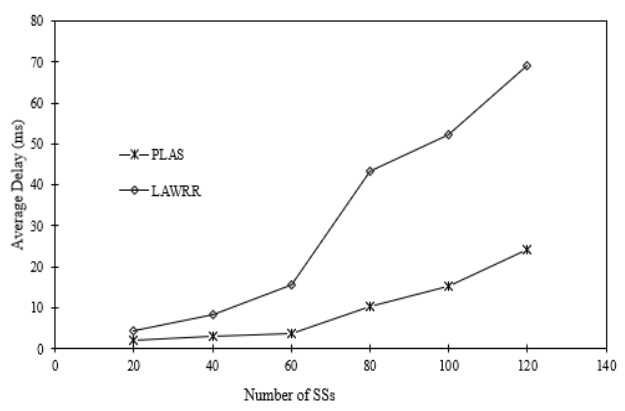

Fig. 4. Average Delay in PLAS and LAWRR for UGS class

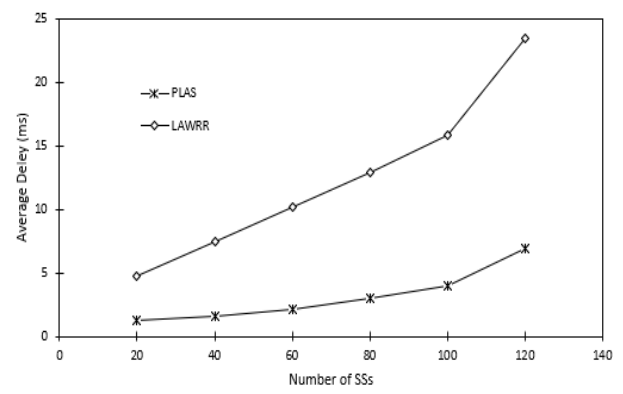

Fig. 5. Average Delay in PLAS and LAWRR for rtPS class

Fig. 4 and 5 show average delay incurred by PLAS \& LAWRR for different SSs for UGS and rtPS (real time) classes respectively. The figures show that PLAS yield better performance than LAWRR in all the experiments. This result is due priority considerations for each class according to QoS requirement, which lead to decrease in the waiting time of packets, and thus reduces average delay in the classes. PLAS reduces delay by about $69.6 \%$ for UGS and about $74.5 \%$ for rtPS.

Fig. 6 shows average throughput achieved by PLAS \& LAWRR for different SSs for UGS class. The figure demonstrates that PLAS outperforms LAWRR. The better performance is due to decrease in delay of queued packets, which causes more packets to be served. PLAS increases throughput of LAWRR by about $32 \%$.

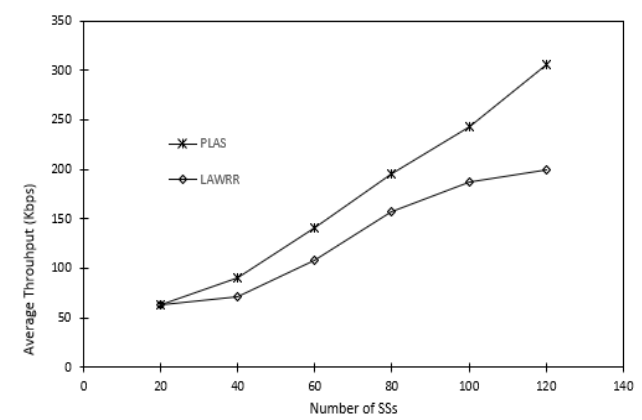

Fig. 6. Average Throughput in PLAS and LAWRR for UGS class

Fig. 7 shows average throughput achieved by PLAS \& LAWRR for different SSs for rtPS class. The figure. reveals that PLAS demonstrates superior performance as compared to LAWRR. The performance is as a result of decrease in delay, which made more packets to be served. PLAS increases throughput of LAWRR by about $11.7 \%$.

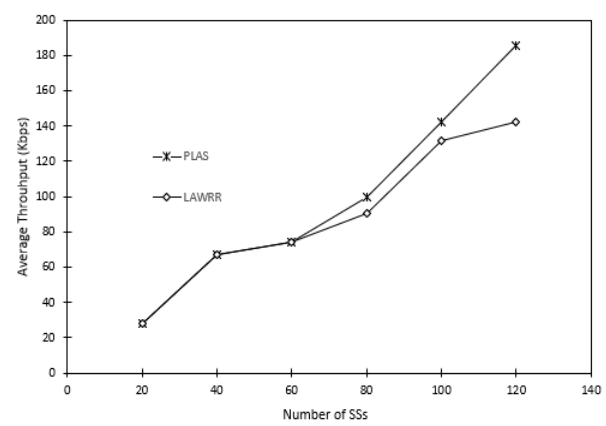

Fig. 7. Average Throuhput in PLAS and LAWRR for rtPS class

\section{CONCLUSION}

In this study, a performance evaluation of PLAS algorithm was conducted against LAWR using ddiscrete event simulation. The experiments were conducted under a more realistic input traffics and additional performance metric for a typical WiMAX network. The traffics are generated from $20,40,60,80,100$ and 120 SSs. The results of the experiments reveal that PLAS reduces delay by about $69.6 \%$ for UGS and about $74.5 \%$ for rtPS. It also increases throughput of LAWRR by $32 \%$ for UGS and $11.7 \%$ for rtPS traffics. The improved performance will lead to better user experience, which will increase number of mobile subscribers and consequently increase revenue for service providers.

\section{ACKNOWLEDGEMENT}

The authors would like to appreciate the Tertiary Education Trust Fund (TETFund) Nigeria for supporting this 
research work through the National Research Fund (NRF) Grant No: CC - STI.

\section{REFERENCES}

[1] K. S. S. Prasad, "An Efficient QoS Scheduling Algorithm for IEEE 802.16 Networks," Middle-East Journal of Scientific Research, vol. 20(10), pp. 1332-1338, 2014.

[2] S. Ibrahim, S. Shamala, J. Azmin, and Z. Zuriati, "A load-aware weighted round robin algorithm for IEEE 802.16 networks," EURASIP Journal on Wireless Communications and Networking, vol. 2014, pp. $1-12,2014$

[3] A. H. Rashwan, H. M. ElBadawy, and H. H. Ali, "Comparative Assessments for Different WiMAX Scheduling Algorithms," Proceedings of the World Congress on Engineering and Computer Science, vol. 1, pp. 20-29, 2009.

[4] A. T. Marcio, and R. G. Paulo, "A new and efficient adaptive scheduling packets for the uplink traffic in WiMAX networks," EURASIP Journal on Wireless Communications and Networking. vol. 113, pp.1-11, 2011

[5] C. So-in, R. Jain, and A. Tamimi, "Scheduling in IEEE 802. 16e Mobile WiMAX networks, "IEEE Journal on Selected Areas in Communications" vol. 27(2), pp. 156-171. 2009.

[6] M. S. Arhaaif, "Comparative Study of Scheduling Algorithms in WiMAX," International Journal of Scientific \& Engineering Research," vol. 2(2), pp. 1-7, 2011.

[7] M. Gidlund, and G. Wang, "Uplink Scheduling Algorithms for QoS Support in Broadband Wireless Access Networks," Journal of communications, vol. 4(2), pp. 133-142, 2009.

[8] A. Jain, and A. K. Verma, "Comparative Study of Scheduling Algorithms for WiMAX," Proceedings of the National Conference on Mobile and Pervasive Computing, Compc 08, Chennai, India. pp. 10 13, 2008.

[9] L. H. Ellen, "Round-Robin Scheduling for Max-Min Fairness in Data Networks," IEEE Journal on Selected Areas in Communications, vol. 9(7), pp. 1024-1039, 1991.

[10] K. Manolis, S. Stefenos, C. Costac, "Weighted Round Robin Cell Multiplexing in a General-Purpose ATM Switch Chip, ” IEEE Journal on Selected Areas in Communications, vol. 9(8), pp. 1265-1279, 1991.

[11] W. Mardini, and M. M. A. Alfoul, "Modified WRR Scheduling Algorithm for WiMAX Networks," International Journal of Network Protocols and Algorithms, vol. 3(2), pp. 24-53, 2011.

[12] B. Mohamed-el-Amin, A. Abdelhafid, and L. Pascal, "Adaptive Scheduling Mechanism for IPTV over WiMAX IEEE 802.16j networks," International Journal of Communication Systems, vol. 23(5), pp. 633-652, 2012.

[13] P. Zuber, and D. Upena, "Design and Implementation of low latency weighted round robin (llwrr) scheduling for high speed networks," International Journal of Wireless \& Mobile networks (IJWMN), vol. 6(4), pp. 59-71, 2014.

[14] A. Mohammed, I. Saidu and A. Abdulazeez, "A Priority Load-Aware Scheduling (PLAS) Algorithm for Wireless Broadband Networks," in Odumuyiwa V., Adegboyega O., and Uwadia C. (eds), e-Infrastructure and e-Services for Developing Countries. AFRICOMM 2017. Lecture Notes of the Institute for Computer Sciences, Social Informatics and Telecommunications Engineering, Springer, Cham, vol. 250, pp. 4959, 2018.

[15] Z. A. Shareef, M. Hussin, A. Abdullah, and M. Abdullah, "Class-based QoS Scheduling of WiMAX networks," Journal of High Speed Networks, vol 24(4), pp. 345-362, 2018. 